University of Nebraska - Lincoln

DigitalCommons@University of Nebraska - Lincoln

Sociology Department, Faculty Publications

Sociology, Department of

8-1-2008

\title{
Statistical Comparison of Residential Soil Concentrations of PCDDs, PCDFs, and PCBs from Two Communities in Michigan
}

\author{
Avery Demond \\ University of Michigan College of Engineering., averyd@umich.edu \\ P. Adriaens \\ University of Michigan College of Engineering \\ T. Towey \\ LimnoTech, Ann Arbor, MI
}

S.-C. Chang

National Chung Hsing University

B. Hong

University of Michigan School of Public Health

See next page for additional authors

Follow this and additional works at: https://digitalcommons.unl.edu/sociologyfacpub

Part of the Sociology Commons

Demond, Avery; Adriaens, P.; Towey, T.; Chang, S.-C.; Hong, B.; Chen, Q.; Chang, C.-W.; Franzblau, A. J.; Garabrant, D.; Gillespie, B.; Hedgeman, E.; Knutson, K.; Lee, C. Y.; Lepkowski, J.; Olson, Kristen M.; Ward, B.; Zwica, L.; Luksemburg, W.; and Maier, M, "Statistical Comparison of Residential Soil Concentrations of PCDDs, PCDFs, and PCBs from Two Communities in Michigan" (2008). Sociology Department, Faculty Publications. 16.

https://digitalcommons.unl.edu/sociologyfacpub/16

This Article is brought to you for free and open access by the Sociology, Department of at DigitalCommons@University of Nebraska - Lincoln. It has been accepted for inclusion in Sociology Department, Faculty Publications by an authorized administrator of DigitalCommons@University of Nebraska - Lincoln. 


\section{Authors}

Avery Demond, P. Adriaens, T. Towey, S.-C. Chang, B. Hong, Q. Chen, C.-W. Chang, A. J. Franzblau, D. Garabrant, B. Gillespie, E. Hedgeman, K. Knutson, C. Y. Lee, J. Lepkowski, Kristen M. Olson, B. Ward, L. Zwica, W. Luksemburg, and M Maier 
The American Chemical Society's policy on archiving permits the posting of only the author names, title, abstract, tables, and figures of articles published and/or copyright by the ACS.

In respect of this policy, other portions of this article have been suppressed.

The complete article may be found on the publisher's website (requires subscription) at:

http://pubs.acs.org/cgi-bin/article.cgi/esthag/2008/42/i15/pdf/es702554g.pdf [PDF file]

or

http://pubs.acs.org/cgi-bin/article.cgi/esthag/2008/42/i15/html/es702554g.html [html text] 
Statistical Comparison of Residential Soil Concentrations of PCDDs, PCDFs, and PCBs from Two Communities in Michigan

A. DEMOND, ${ }^{*}{ }^{+}$P. ADRIAENS,$^{+}$

T. TOWEY, ${ }^{\prime}$ S. - C. CHANG, \$ B. HONG, "

Q. CHEN, ${ }^{\perp} \mathrm{C}$. - W. CHANG,"

A. FRANZBLAU," D. GARABRAN T, "

B. GILLESPIE, ${ }^{\perp}$ E. HEDGEMAN,"

K. KNUTSON, "C. Y. LEE, ${ }^{\perp}$

J. LEPKOWSKI, " $\mathrm{K}$. OLSON, " B. WARD, "

L. ZWICA," W. LUKSEMBURG, ' AND

M. M A IE R -

Department of Civil and Environmental Engineering, University of Michigan College of Engineering, Ann Arbor, Michigan 48109, LimnoTech, 501 Avis Drive, Ann Arbor, Michigan 48108, Department of Environmental Engineering, National Chung Hsing University, Taichung 402, Taiwan, Department of Environmental Health Sciences, University of Michigan School of Public Health, Ann Arbor, Michigan 48109, Department of Biostatistics, University of Michigan School of Public Health, Ann Arbor, Michigan 48109, Institute for Social Research, University of Michigan Ann Arbor, Michigan 48109, and Vista Analytical Laboratory, 1100 Windfield Way, El Dorado Hills, California 95762

Received October 10, 2007. Revised manuscript received April 22, 2008. Accepted April 24, 2008.

The University of Michigan dioxin exposure study was undertaken to address concerns that the industrial discharge of dioxin-like compounds in the Midland, $\mathrm{Ml}$ area had resulted in contamination of soils in the Tittabawassee River floodplain and downwind of the incinerator. The study was designed in a rigorously statistical manner comprising soil measurements of 29 polychlorinated dibenzo- $p$-dioxins (PCDDs), polychlorinated dibenzofurans (PCDFs), and polychlorinated biphenyls (PCBs) from 766 residential properties, selected probabilistically, in the Midland area and in Jackson and Calhoun Counties (Michigan) as a background comparison. A statistical comparison determined that the geometric mean toxic equivalent (TEO) levels in samples from the target populations were statistically significantly above background. In addition, the probabilities of being above the 75th and 95th percentiles of background were also greater. Congener contributions to the TEO were dominated by 2,3,4,7,8-PeCDF and 2,3,7,8-TCDF in the floodplain and by $2,3,7,8-T C D D$ in the incinerator plume. However, PCB 126 was the top congener contributing to the background TEQ.

* Corresponding author phone: (734) 763-3708; fax: (734) 7632275; e-mail: averyd@umich.edu.

${ }^{\dagger}$ University of Michigan College of Engineering.

${ }^{\ddagger}$ LimnoTech.

${ }^{\S}$ National Chung Hsing University.

" Department of Environmental Health Sciences, University of Michigan School of Public Health.

${ }^{\perp}$ Department of Biostatistics, University of Michigan School of Public Health.

" University of Michigan Ann Arbor.

- Vista Analytical Laboratory.
On the basis of statistical inference to the total population, it was estimated that about $36 \%$ of the properties in the floodplain and incinerator plume have at least one soil sample over the Michigan Department of Environmental Quality's soil direct contact criterion of $90 \mathrm{pg} / \mathrm{g}$ TEQ. 
TABLE 1. Number of Samples, Arithmetic Mean TEQ, Standard Error (in Parentheses), Median, 75th and 95th Percentiles (in Italics), and Range for Each Soil Composite Type for the Five Areas (in pg/g)

\begin{tabular}{|c|c|c|c|c|c|}
\hline (A) $\mathrm{TEQ}_{\mathrm{DFP}-2005}$ & HP $0-1$ in. & HP 1-6 in. & garden & NR $0-1$ in. & NR 1-6 in. \\
\hline floodplain & $\begin{array}{l}N=203 \\
56.5(9.7) \\
11.4,35.4,223.1 \\
1.1-1881.4\end{array}$ & $\begin{array}{l}N=198 \\
56.2(11.1) \\
10.3,25.6,214.4 \\
1.1-2164.7\end{array}$ & $\begin{array}{l}N=132 \\
50.7(11.0) \\
9.1,31.5,218.4 \\
1.6-2199.3\end{array}$ & $\begin{array}{l}N=191 \\
238.5(40.5) \\
14.5,242.5,1041.8 \\
1.1-7258.2\end{array}$ & $\begin{array}{l}N=191 \\
286.6(52.3) \\
11.8,298.5,1242.3 \\
0.7-5356.8\end{array}$ \\
\hline plume & $\begin{array}{l}N=37 \\
109.2(31.0) \\
58.2,111.9,257.2 \\
6.3-745.5\end{array}$ & $\begin{array}{l}N=36 \\
101.1(35.9) \\
54.2,70.8,251.1 \\
7.0-849.9\end{array}$ & $\begin{array}{l}N=27 \\
64.0(15.1) \\
53.8,59.7,323.9 \\
19.6-323.9\end{array}$ & NA & NA \\
\hline near floodplain & $\begin{array}{l}N=164 \\
52.0(36.7) \\
3.9,10.4,102.9 \\
0.8-2299.8\end{array}$ & $\begin{array}{l}N=75 \\
64.7(28.3) \\
11.2,36.8,306.3 \\
1.3-705.5\end{array}$ & $\begin{array}{l}N=100 \\
20.4(4.9) \\
5.4,11.6,90.5 \\
0.7-547.0\end{array}$ & NA & NA \\
\hline other & $\begin{array}{l}N=168 \\
13.5(2.0)\end{array}$ & $\begin{array}{l}N=87 \\
114.3(99.6)\end{array}$ & $\begin{array}{l}N=101 \\
9.8(1.0)\end{array}$ & NA & NA \\
\hline Midland/Saginaw & $\begin{array}{l}5.3,13.2,59.4 \\
0.8-157.7\end{array}$ & $\begin{array}{l}11.1,18.2,64.6 \\
1.1-11219.1\end{array}$ & $\begin{array}{l}4.2,12.1,37.3 \\
0.4-89.4\end{array}$ & & \\
\hline Jackson/Calhoun & $\begin{array}{l}N=194 \\
6.9(0.8) \\
3.6,7.6,22.6 \\
0.4-186.2\end{array}$ & $\begin{array}{l}N=53 \\
11.2(1.1) \\
8.8,13.2,29.9 \\
0.9-35.3\end{array}$ & $\begin{array}{l}N=124 \\
4.9(1.4) \\
2.5,5.2,14.5 \\
0.3-72.8\end{array}$ & NA & NA \\
\hline (B) $\mathrm{TEQ}_{\mathrm{DF}-2005}$ & HP $0-1$ in. & HP 1-6 in. & garden & NR $0-1$ in. & NR 1-6 in. \\
\hline floodplain & $\begin{array}{l}N=203 \\
55.7(9.7) \\
10.6,35.1,220.0 \\
1.0-1878.3\end{array}$ & $\begin{array}{l}N=198 \\
55.4(11.1) \\
9.9,24.4,213.9 \\
1.0-2159.6\end{array}$ & $\begin{array}{l}N=132 \\
49.5(11.0) \\
8.8,31.2,217.7 \\
1.0-2197.5\end{array}$ & $\begin{array}{l}N=191 \\
237.2(40.4) \\
12.7,240.5,1038.3 \\
1.0-7242.0\end{array}$ & $\begin{array}{l}N=191 \\
285.0(52.2) \\
11.2,295.8,1231.7 \\
0.7-5335.3\end{array}$ \\
\hline plume & $\begin{array}{l}N=37 \\
103.3(30.6) \\
57.6,96.1,245.7 \\
4.5-742.9\end{array}$ & $\begin{array}{l}N=36 \\
97.6(35.7) \\
53.3,69.9,249.3 \\
6.8-847.5\end{array}$ & $\begin{array}{l}N=27 \\
60.1(12.1) \\
53.0,58.7,260.9 \\
19.3-260.9\end{array}$ & NA & NA \\
\hline near floodplain & $\begin{array}{l}N=164 \\
51.7(36.7) \\
3.6,10.1,102.7 \\
0.8-2298.6\end{array}$ & $\begin{array}{l}N=75 \\
64.3(28.3) \\
11.0,32.7,305.4 \\
1.1-704.8\end{array}$ & $\begin{array}{l}N=100 \\
20.1(4.9) \\
5.0,11.5,90.3 \\
0.7-546.7\end{array}$ & NA & NA \\
\hline other & $\begin{array}{l}N=168 \\
12.8(2.0)\end{array}$ & $\begin{array}{l}N=87 \\
113.4(99.5)\end{array}$ & $\begin{array}{l}N=101 \\
9.3(1.0)\end{array}$ & NA & NA \\
\hline Midland/Saginaw & $\begin{array}{l}4.5,11.9,56.7 \\
0.8-157.4\end{array}$ & $\begin{array}{l}10.1,17.2,64.1 \\
1.0-11217.4\end{array}$ & $\begin{array}{l}3.7,11.1,36.8 \\
0.4-88.8\end{array}$ & & \\
\hline Jackson/Calhoun & $\begin{array}{l}N=194 \\
4.8(0.5) \\
2.9,5.7,12.2 \\
0.3-64.1\end{array}$ & $\begin{array}{l}N=53 \\
8.1(1.2) \\
6.8,8.7,22.2 \\
0.7-31.9\end{array}$ & $\begin{array}{l}N=124 \\
3.1(0.3) \\
2.0,4.0,9.4 \\
0.2-18.5\end{array}$ & NA & NA \\
\hline (C) TEO ${ }_{\text {DFP-1998 }}$ & HP $0-1$ in. & HP 1-6 in. & garden & NR $0-1$ in. & NR 1-6 in. \\
\hline floodplain & $\begin{array}{l}N=203 \\
72.2(12.7) \\
12.6,41.6,294.0 \\
1.2-2483.5\end{array}$ & $\begin{array}{l}N=198 \\
71.9(14.6) \\
11.8,30.8,276.5 \\
1.2-2841.1\end{array}$ & $\begin{array}{l}N=132 \\
64.4(14.5) \\
10.3,40.4,250.1 \\
1.8-2951.8\end{array}$ & $\begin{array}{l}N=191 \\
302.1(52.1) \\
15.0,283.3,1329.6 \\
1.2-9351.0\end{array}$ & $\begin{array}{l}N=191 \\
363.4(67.5) \\
12.6,393.0,1469.0 \\
0.8-6758.9\end{array}$ \\
\hline plume & $\begin{array}{l}N=37 \\
121.2(38.7) \\
59.2,114.1,270.7 \\
6.9-925.8\end{array}$ & $\begin{array}{l}N=36 \\
114.4(45.5) \\
58.0,71.4,294.7 \\
7.1-1070.9\end{array}$ & $\begin{array}{l}N=27 \\
68.4(17.1) \\
54.7,62.5,363.1 \\
19.9-363.1\end{array}$ & NA & NA \\
\hline near floodplain & $\begin{array}{l}N=164 \\
67.8(48.7) \\
4.3,11.0,135.3 \\
0.9-3055.9\end{array}$ & $\begin{array}{l}N=75 \\
83.2(36.9) \\
12.1,47.8,403.7 \\
1.3-919.4\end{array}$ & $\begin{array}{l}N=100 \\
25.3(6.5) \\
6.0,12.6,117.6 \\
0.8-730.3\end{array}$ & NA & NA \\
\hline
\end{tabular}




\section{TABLE 1. Continued}

(C) TEQ ${ }_{\text {DFP-1998 }}$

other

$N=168$

$15.6(2.4)$

Midland/Saginaw

$5.5,14.6,74.3$

$0.9-205.0$

$$
N=194
$$

Jackson/Calhoun
HP 1-6 in.

$N=87$

$152.9(135.8)$

$12.9,22.5,83.7$

$1.1-15308.7$

$N=53$

$13.2(1.4)$

$1.0-45.1$
$10.3,14.5,35.5$ garden

$N=101$

10.7 (1.1)

$4.5,12.6,37.9$

$0.4-115.9$

$N=124$

$5.5(1.6)$

2.7, $5.7,14.8$

$0.3-87.9$
NR 0-1 in. NR 1-6 in.

NA

NA

$0.4-329.7$

${ }^{a}$ TEQ ${ }_{\text {DFP-2005 }}$ is calculated using the 29 PCDD, PCDF, and PCB congeners specified by the WHO and the 2005 TEFs (14); TEQ DF-2005 is calculated using the 17 PCDD and PCDF congeners specified by the WHO and the 2005 TEFs (14); TEQ DFP-1998 is calculated using the 29 PCDD, PCDF, and PCB congeners specified by the WHO and the 1998 TEFs (6). HP = house perimeter; $\mathrm{NA}=$ not applicable; $\mathrm{NR}=$ near river.

TABLE 2. Statistics for Pair-Wise Comparisons of TEQ $\mathrm{DFP}_{2005}$ for HP 0-1 Inch Soil Composites ${ }^{d}$

\begin{tabular}{|c|c|c|c|}
\hline area & $\begin{array}{c}\text { geometric } \\
\text { mean } \\
\text { TEQ }_{\text {DFP-2005 }} \\
(\mathbf{p g} / \mathrm{g})\end{array}$ & $\begin{array}{c}\text { percent } \\
\text { above } \\
7.6 \mathrm{pg} / \mathrm{g}^{\mathrm{a}}\end{array}$ & $\begin{array}{c}\text { percent } \\
\text { above } \\
22.6 \mathrm{pg} / \mathrm{g}^{b}\end{array}$ \\
\hline floodplain & 15.7 & 68 & 31 \\
\hline plume & 73 & 98 & 97 \\
\hline near floodplain & 5.9 & 33 & 14 \\
\hline other $\mathrm{M} / \mathrm{S}$ & 6.6 & 41 & 15 \\
\hline Jackson/Calhoun & 3.5 & $24^{c}$ & 5 \\
\hline
\end{tabular}

${ }^{a} \mathrm{TEQ}_{\mathrm{DFP}-2005}=7.6 \mathrm{pg} / \mathrm{g}$ is the 75 th percentile of the Jackson/Calhoun distribution. ${ }^{b}$ TEQ $_{\text {DFP-2005 }}=22.6 \mathrm{pg} / \mathrm{g}$ is the 95th percentile of the Jackson/Calhoun distribution. ${ }^{c}$ The 75th percentile for Jackson/Calhoun is actually 7.5800 $\mathrm{pg} / \mathrm{g}$, which was rounded to $7.6 \mathrm{pg} / \mathrm{g}$ for the purposes of the Wald chi-square test. Because of the multiple values between 7.5800 and 7.600 , actually $24.07 \%$ is above 7.600 $\mathrm{pg} / \mathrm{g}$ in Jackson/Calhoun. ${ }^{d} \mathrm{HP}=$ house perimeter; $\mathrm{J} / \mathrm{C}=$ Jackson/Calhoun; M/S = Midland Saginaw. 
TABLE 3. p-Values for Pair-Wise Comparisons of $\mathrm{TEO}_{\text {DFP-2005 }}$ Among the HP 0-1 Inch Soil Composites from the Five Areas ${ }^{a}$

\begin{tabular}{clrrrr}
\multicolumn{1}{c}{ area } & plume & near floodplain & other M/S & Jackson/ Calhoun \\
& geometric mean & $<0.0001-$ & $<0.0001+$ & $<0.0001+$ & $<0.0001+$ \\
floodplain & odds $>7.6 \mathrm{pg} / \mathrm{g}$ & $0.0015-$ & $<0.0001+$ & $<0.0001+$ & $<0.0001+$ \\
& odds $>22.6 \mathrm{pg} / \mathrm{g}$ & $<0.0001-$ & $0.0016+$ & $0.0043+$ & $<0.0001+$ \\
& geometric mean & & $<0.0001+$ & $<0.0001+$ & $<0.0001+$ \\
plume & odds $>7.6 \mathrm{pg} / \mathrm{g}$ & & $<0.0001+$ & $<0.0001+$ & $<0.0001+$ \\
& odds $>22.6 \mathrm{pg} / \mathrm{g}$ & & $<0.0001+$ & $<0.0001+$ & $<0.0001+$ \\
& geometric mean & & & $0.6162-$ & $0.0098+$ \\
near FP & odds $>7.6 \mathrm{pg} / \mathrm{g}$ & & & $0.2702-$ & $0.1378+$ \\
& odds $>22.6 \mathrm{pg} / \mathrm{g}$ & & & $0.8480-$ & $0.0112+$ \\
& geometric mean & & & & $<0.0001+$ \\
other $\mathrm{M} / \mathrm{S}$ & odds $>7.6 \mathrm{pg} / \mathrm{g}$ & & & & $0.0018+$ \\
& odds $>22.6 \mathrm{pg} / \mathrm{g}$ & & & & $0.0075+$
\end{tabular}

${ }^{a} \mathrm{HP}=$ house perimeter; FP = floodplain; M/S = Midland/Saginaw. Geometric means were compared using a $t$ test, and the results are given in the first row for each area. The probabilities of being above $7.6 \mathrm{pg} / \mathrm{g}$ [Jackson/Calhoun's 75 th percentile] or $22.6 \mathrm{pg} / \mathrm{g}$ [Jackson/Calhoun's 95th percentile] were compared using a Wald chi test, and the results are given in the second and third rows, respectively, for each area. $p$-values of $<|0.05|$ indicate statistical significance. " + " after the $p$-value indicates that the samples from the area listed in the row have a higher geometric mean or a higher probability of being above the cutpoints than the samples from the area listed in the column. "-" after the $p$-value indicates that the samples from the area listed in the row have a lower mean or a lower probability of being above the cutpoint than the samples from the area listed in the column.

\section{TABLE 4. Estimate of Percentages of Properties with at Least One Soil Composite ${ }^{a}$ Over TEQ $=90 \mathrm{pg} / \mathrm{g}$ or Over TEQ $=1000 \mathrm{pg} / \mathrm{g}$}

\begin{tabular}{|c|c|c|c|c|c|c|}
\hline \multirow[b]{2}{*}{ area } & \multicolumn{2}{|c|}{ TEO $_{\text {DFP-2005 }}(\mathrm{pg} / \mathrm{g})$} & \multicolumn{2}{|c|}{ TEO $_{\mathrm{DF}-2005}(\mathrm{pg} / \mathrm{g})$} & \multicolumn{2}{|c|}{ TEO $_{\text {DFP-1998 }}(\mathrm{pg} / \mathrm{g})$} \\
\hline & over 90 & over 1000 & over 90 & over 1000 & over 90 & over 1000 \\
\hline floodplain & 36.5 & 7.0 & 36.5 & 7.0 & 38.0 & 10.7 \\
\hline plume & 35.8 & 0.0 & 35.8 & 0.0 & 38.1 & 4.2 \\
\hline near floodplain & 9.7 & 1.6 & 9.7 & 1.6 & 10.8 & 1.6 \\
\hline other $\mathrm{M} / \mathrm{S}$ & 1.7 & 0.4 & 1.7 & 0.4 & 2.4 & 0.4 \\
\hline Jackson/Calhoun & 0.3 & 0.0 & 0.0 & 0.0 & 1.8 & 0.0 \\
\hline
\end{tabular}

${ }^{a}$ The soil composite may be any of the five types of composites. TEQ $\mathrm{DFP}_{2005}$ is calculated using the 29 PCDD, PCDF, and PCB congeners specified by the WHO and the 2005 TEFs (14); TEQDF-2005 is calculated using the 17 PCDD and PCDF congeners specified by the WHO and the 2005 TEFs (14); TEQDFP-1998 is calculated using the 29 PCDD, PCDF, and PCB congeners specified by the WHO and the 1998 TEFs (6). M/S = Midland/Saginaw. 

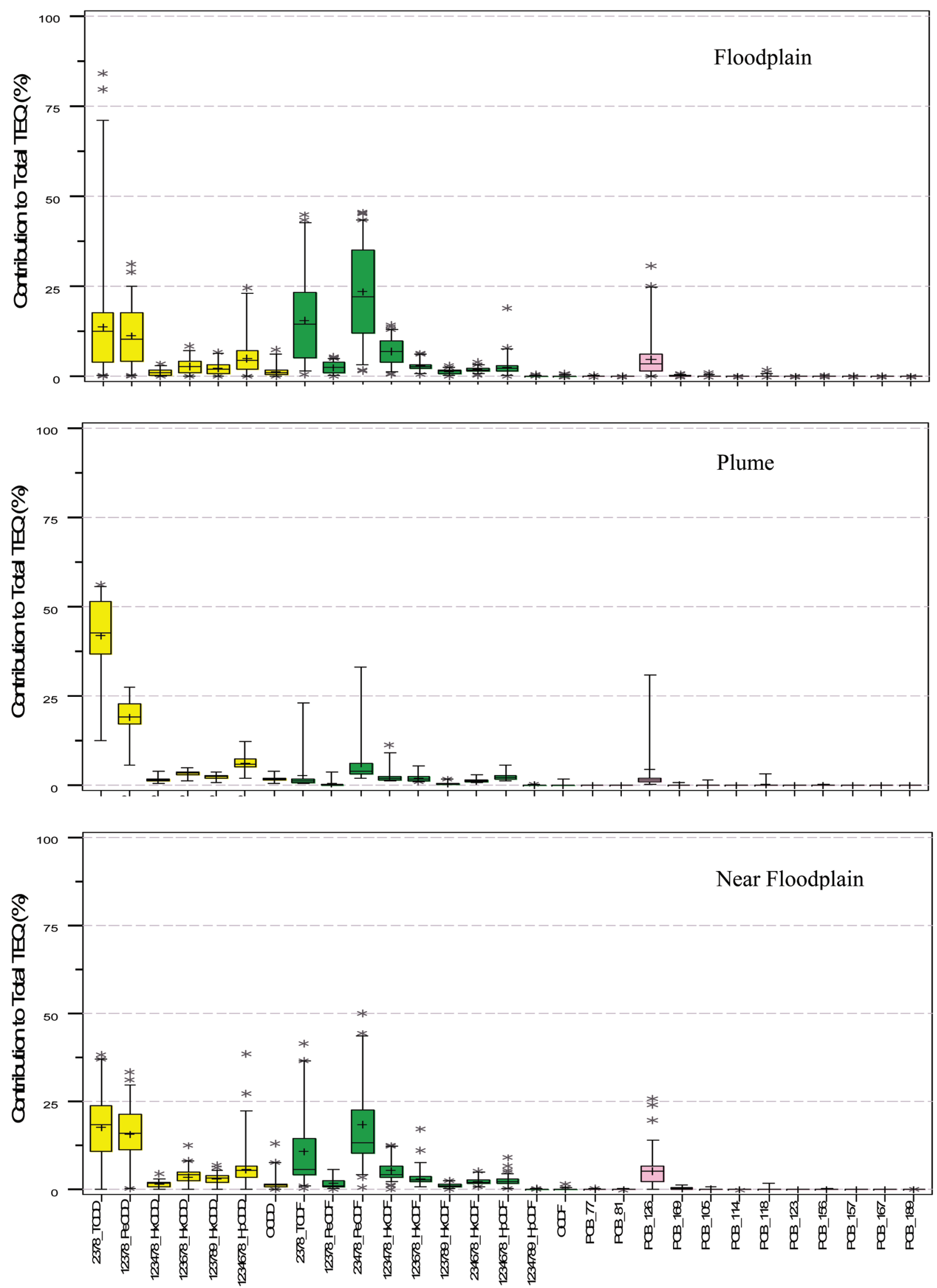

FIGURE 1 

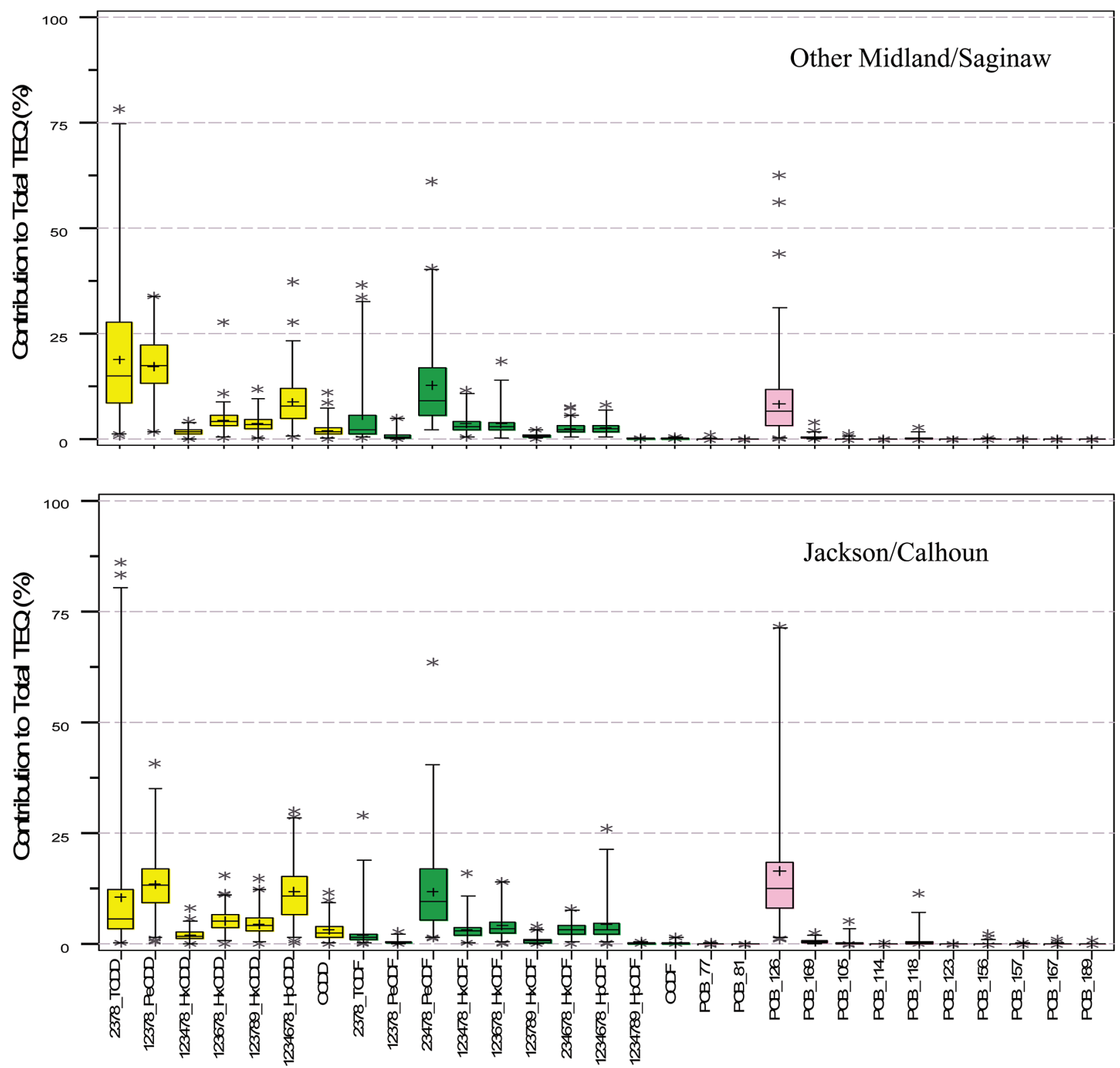

FIGURE 1. Contribution to $\mathrm{TEO}_{\mathrm{DFP}-2005}$ of the individual congeners for the HP $0-1$ in. samples. The plus sign indicates the arithmetic mean. The horizontal line across the box indicates the 50th percentile (median), the lower and upper margins of the box indicate the 25th percentile and 75th percentile, respectively; the upper ticked line extends to the 99th percentile, and the lower ticked line extends to the 1st percentile. The stars show the values above the 99th percentile and below the 1st percentile. 


\section{Acknowledgments}

Financial support for this study comes from the Dow Chemical Company through an unrestricted grant to the University of Michigan. The authors are grateful to Drs. Linda Birnbaum, Paolo Boffetta, Ronald Hites, David Kleinbaum, and Marie Haring Sweeney for their guidance as members of UMDES's Scientific Advisory Board.

\section{Supporting Information Available}

The Supporting Information contains a map of the study area (Figure S1), a diagram of sampling locations at each residence (Figure S2), an example histogram of the measured TEQ ${ }_{\text {DFP-2005 }}$ showing the skewness of the distribution (Figure S3), and a table giving the individual concentrations of the 29 congeners (Table S1). This information is available free of charge via the Internet at http://pubs.acs.org. 
Supporting Information for the manuscript entitled

Statistical Comparison of Residential Soil Concentrations of PCDDs, PCDFs and PCBs from Two Communities in Michigan, USA

Demond, A, Adriaens, P, Towey, T, Chang, S-C, Hong B, Chen, Q, Chang, C-W, Franzblau, A, Garabrant D, Gillespie, B, Hedgeman E, Knutson, K, Lee, CY, Lepkowski, J, Olson, K, Ward, B, Zwica L, Luksemburg W, Maier M

This Supporting Information comprises three figures and one table: a map of the study area (Figure S1), a diagram of sampling locations at each residence (Figure S2), an example histogram of the measured TEQDFP-2005 showing the skewness of the distribution (Figure S3) and a table giving the individual concentrations of the 29 PCDD, PCDF and PCB congeners measured in the study (Table S1). 


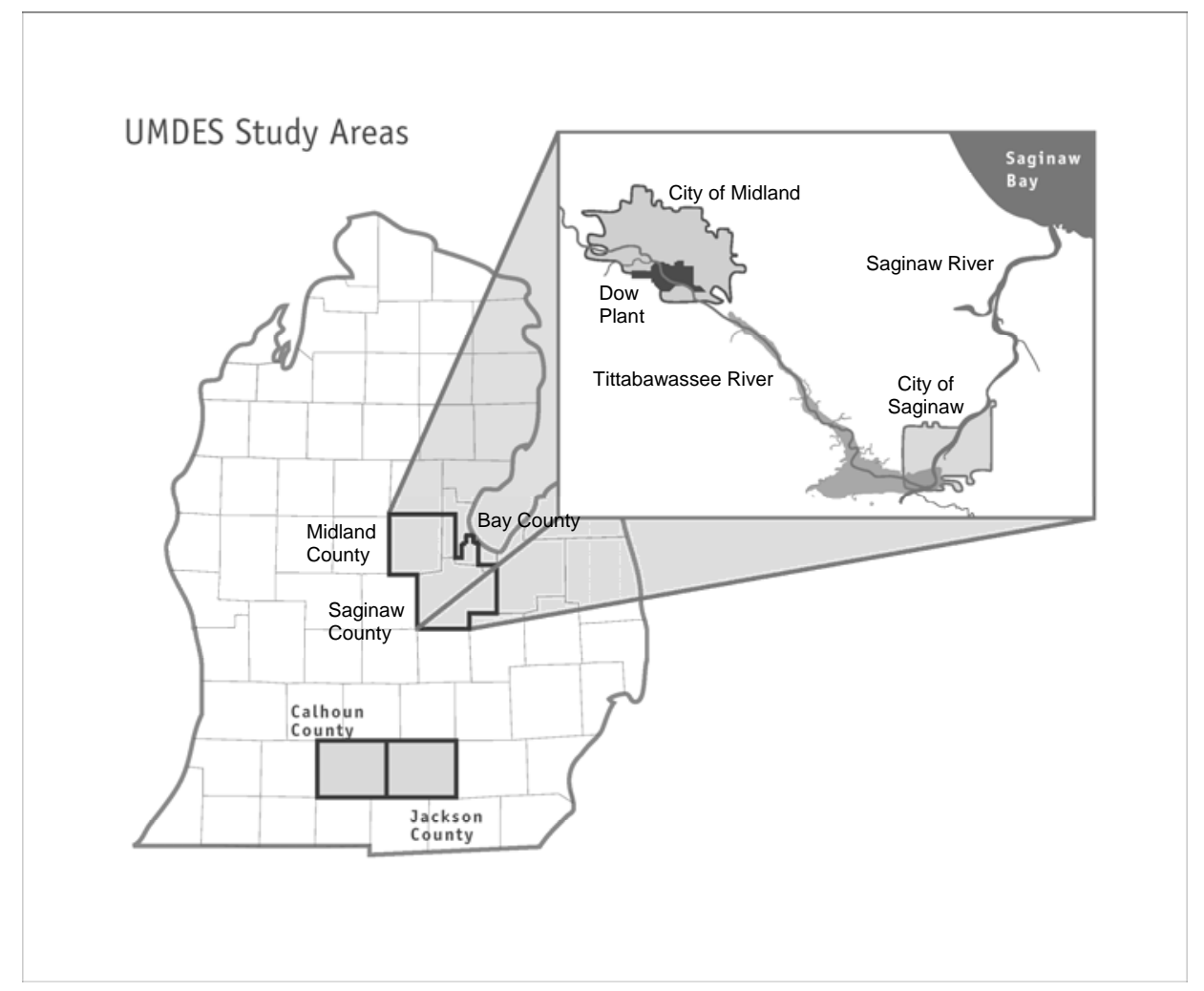

Figure S1. Map of the lower peninsula of the State of Michigan showing the UMDES study areas. Shaded area along Tittabawassee River shows the river's 100-year FEMA floodplain. The floodplain is very narrow until it approaches the City of Saginaw. 


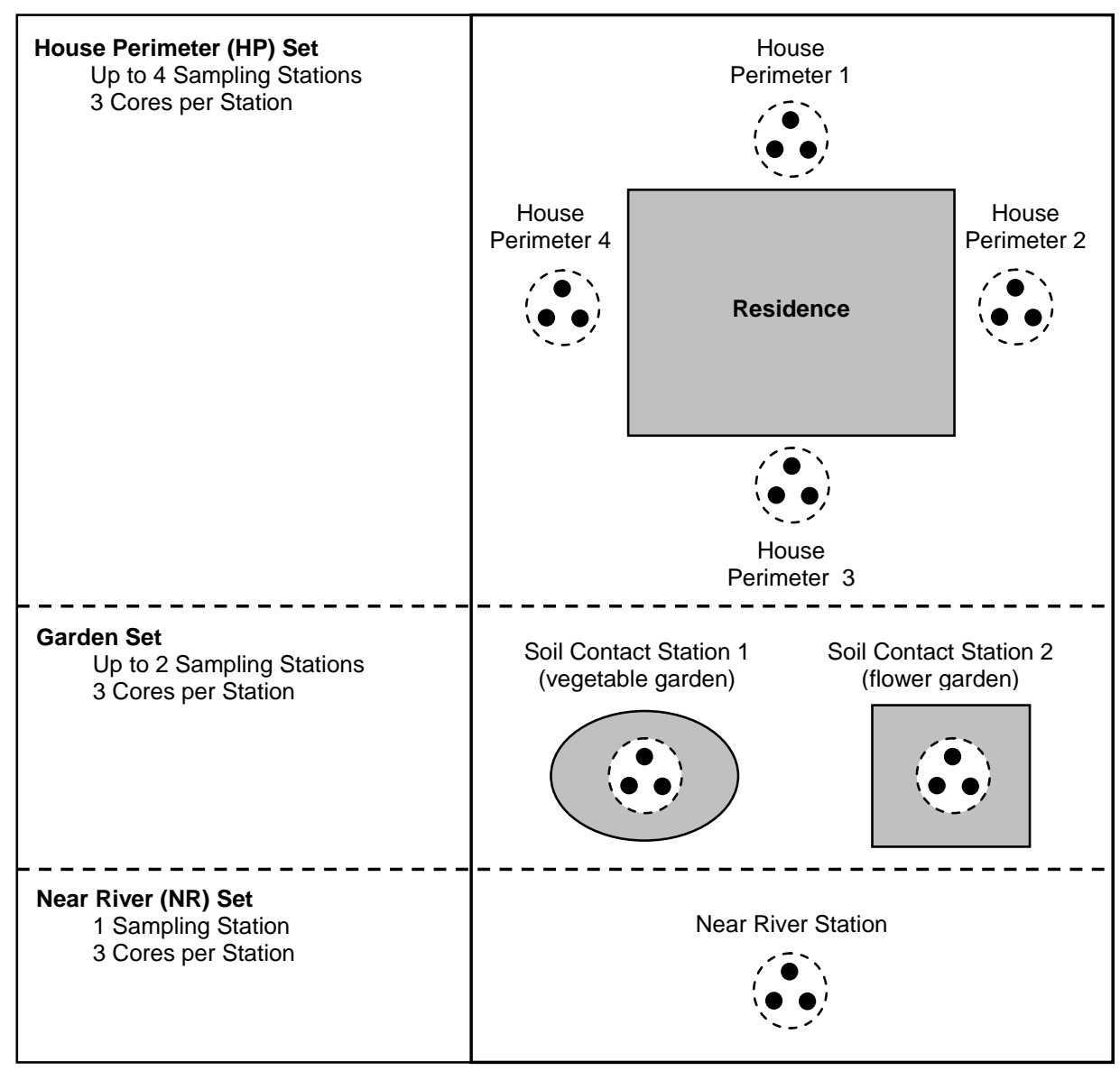

Figure S2. Location of sampling stations at residences. The dotted circles represent the sampling stations. The small dark circles represent the individual cores. 


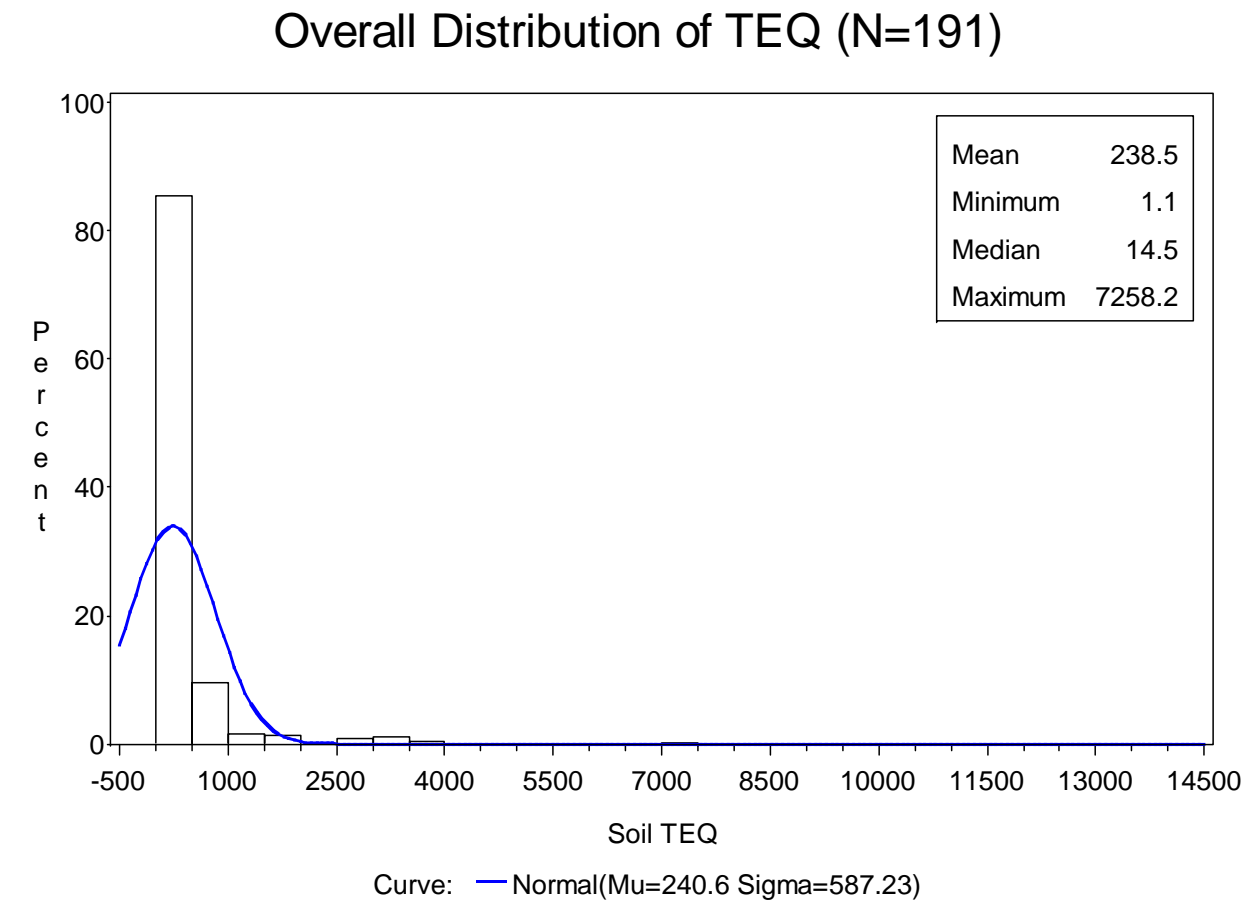

Figure S3. Histogram of TEQ ${ }_{D F}-2005$ for Near River 0-1 inch soil samples showing the skewness of the distribution. 
Table S1. Mean Concentration, Standard Error (in parentheses), Median, $75^{\text {th }}$ and $95^{\text {th }}$ Percentiles (in italics), Number of Samples Below LOD and Median LOD [in brackets] for Each Congener for HP 0-1 Inch Soil Composites for the Five Areas (in pg/g without TEQ adjustment)

\begin{tabular}{|c|c|c|c|c|c|}
\hline $\begin{array}{l}\text { Mean (Standard Error) } \\
\text { Median, 75\%ile, 95\%ile } \\
\text { \# < LOD [Median LOD] }\end{array}$ & Floodplain & Plume & $\begin{array}{c}\text { Near } \\
\text { Floodplain }\end{array}$ & Other M/S & $\begin{array}{l}\text { Jackson/ } \\
\text { Calhoun }\end{array}$ \\
\hline $2,3,7,8-\mathrm{TCDD}$ & $\begin{array}{c}2.1(0.3) \\
1.3,2.3,5.3 \\
11[0.4] \\
\end{array}$ & $\begin{array}{c}36.6(5.0) \\
32.1,46.3,93.0 \\
0[\mathrm{NA}]\end{array}$ & $\begin{array}{c}1.0(0.1) \\
0.7,1.2,3.2 \\
24[0.4] \\
\end{array}$ & $\begin{array}{c}2.5(0.7) \\
0.9,1.8,7.6 \\
24[0.3]\end{array}$ & $\begin{array}{c}0.9(0.2) \\
0.2,0.3,2.6 \\
125[0.2] \\
\end{array}$ \\
\hline 1,2,3,7,8-PeCDD & $\begin{array}{c}1.6(0.1) \\
1.1,2.0,4.7 \\
11[0.8] \\
\end{array}$ & $\begin{array}{c}16.0(2.1) \\
11.1,19.3,41.9 \\
0 \text { [NA] }\end{array}$ & $\begin{array}{c}1.0(0.2) \\
0.7,1.0,2.7 \\
16[0.4] \\
\end{array}$ & $\begin{array}{c}1.5(0.2) \\
0.9,1.5,5.0 \\
13[0.5] \\
\end{array}$ & $\begin{array}{c}0.7(0.1) \\
0.4,0.8,1.6 \\
45[0.2]\end{array}$ \\
\hline 1,2,3,4,7,8-HxCDD & $\begin{array}{c}1.5(0.1) \\
1.1,1.9,3.8 \\
13[0.7]\end{array}$ & $\begin{array}{c}12.7(1.8) \\
10.0,14.3,36.6 \\
0[\mathrm{NA}]\end{array}$ & $\begin{array}{c}1.0(0.1) \\
0.6,1.0,2.6 \\
14[0.4]\end{array}$ & $\begin{array}{c}1.4(0.1) \\
1.0,1.5,3.9 \\
8[0.4] \\
\end{array}$ & $\begin{array}{c}0.9(0.1) \\
0.6,1.0,2.7 \\
25[0.2]\end{array}$ \\
\hline 1,2,3,6,7,8-HxCDD & $\begin{array}{c}4.4(0.4) \\
2.9,4.8,11.7 \\
0[\mathrm{NA}] \\
\end{array}$ & $\begin{array}{c}29.7(4.7) \\
20.6,38.2,87.6 \\
0[\mathrm{NA}] \\
\end{array}$ & $\begin{array}{c}3.0(0.7) \\
1.4,2.6,5.6 \\
3[0.7] \\
\end{array}$ & $\begin{array}{c}3.7(0.3) \\
2.4,4.1,11.6 \\
0 \text { [NA] }\end{array}$ & $\begin{array}{c}2.8(0.3) \\
1.8,3.2,7.5 \\
10[0.3] \\
\end{array}$ \\
\hline 1,2,3,7,8,9-HxCDD & $\begin{array}{c}3.0(0.3) \\
2.1,3.4,7.5 \\
3[1.4]\end{array}$ & $\begin{array}{c}20.3(2.8) \\
14.8,24.0,56.2 \\
0[\mathrm{NA}]\end{array}$ & $\begin{array}{c}1.9(0.2) \\
1.2,1.9,4.4 \\
3[0.8]\end{array}$ & $\begin{array}{c}2.8(0.2) \\
1.9,3.4,7.4 \\
1[0.6]\end{array}$ & $\begin{array}{c}2.0(0.2) \\
1.4,, 2.4,5.6 \\
9[0.5]\end{array}$ \\
\hline 1,2,3,4,6,7,8-HpCDD & $\begin{array}{c}87.6(10.7) \\
50.5,95.2,226 \\
0[\mathrm{NA}]\end{array}$ & $\begin{array}{c}532.1(76.3) \\
370.0,705.5,1410 \\
0 \text { [NA] }\end{array}$ & $\begin{array}{c}63.6(20.7) \\
21.7,48.2,135 \\
0 \text { [NA] }\end{array}$ & $\begin{array}{c}76.0(6.4) \\
43.8,92.9,259.0 \\
0 \text { [NA] }\end{array}$ & $\begin{array}{c}65.0(7.8) \\
36.2,78.2,212 \\
0[\mathrm{NA}] \\
\end{array}$ \\
\hline OCDD & $\begin{array}{c}792.3(107.6) \\
423.5,886,2110 \\
0[\mathrm{NA}] \\
\end{array}$ & $\begin{array}{c}4943.8(708.9) \\
3560,6770,11800 \\
0 \text { [NA] } \\
\end{array}$ & $\begin{array}{c}533.4(148.6) \\
158,419,1360 \\
0[\mathrm{NA}] \\
\end{array}$ & $\begin{array}{c}677.0(69.1) \\
333.0,766.0,2430 \\
0 \text { [NA] } \\
\end{array}$ & $\begin{array}{c}592.0(82.3) \\
274,728,1700 \\
0 \text { [NA] } \\
\end{array}$ \\
\hline 2,3,7,8-TCDF & $\begin{array}{c}167.7(35.2) \\
14.4,65.5,736 \\
0[\mathrm{NA}]\end{array}$ & $\begin{array}{c}90.4(74.5) \\
6.8,18.3,333.0 \\
0[\mathrm{NA}]\end{array}$ & $\begin{array}{c}150.4(113.1) \\
1.9,10.0,328 \\
1[1.1]\end{array}$ & $\begin{array}{c}19.0(5.3) \\
1.1,5.2,120 \\
19[0.5]\end{array}$ & $\begin{array}{c}1.1(0.1) \\
0.5,1.4,2.8 \\
31[0.3]\end{array}$ \\
\hline 1,2,3,7,8-PeCDF & $\begin{array}{c}78.8(15.2) \\
7.4,36.0,337.5 \\
1[0.3] \\
\end{array}$ & $\begin{array}{l}51.2(40.8) \\
5.5,13.5,131.0 \\
0[\mathrm{NA}] \\
\end{array}$ & $\begin{array}{c}90.0(71.1) \\
1.4,6.7,174 \\
3[0.3]\end{array}$ & $\begin{array}{c}9.9(2.5) \\
0.9,3.9,72.6 \\
23[0.4]\end{array}$ & $\begin{array}{c}0.9(0.1) \\
0.4,1.1,2.9 \\
40[0.2]\end{array}$ \\
\hline 2,3,4,7,8-PeCDF & $\begin{array}{c}70.9(13.7) \\
7.6,30.7,322 \\
1[0.9]\end{array}$ & $\begin{array}{c}49.6(35.4) \\
7.6,20.4,117.0 \\
0[\mathrm{NA}]\end{array}$ & $\begin{array}{c}70.0(53.3) \\
1.6,6.4,145 \\
2[0.3]\end{array}$ & $\begin{array}{c}9.7(2.1) \\
1.4,4.8,66.8 \\
7[1.0]\end{array}$ & $\begin{array}{c}2.4(0.3) \\
1.0,3.0,7.8 \\
13[0.2]\end{array}$ \\
\hline 1,2,3,4,7,8-HxCDF & $\begin{array}{c}53.6(9.5) \\
6.5,25.7,229 \\
0[\mathrm{NA}]\end{array}$ & $\begin{array}{c}47.9(29.3) \\
13.2,20.9,199.0 \\
0[\mathrm{NA}]\end{array}$ & $\begin{array}{c}56.9(44.0) \\
1.8,5.3,112 \\
0 \text { [NA] }\end{array}$ & $\begin{array}{c}7.1(1.5) \\
1.4,4.5,34.8 \\
2[0.5]\end{array}$ & $\begin{array}{c}2.0(0.2) \\
1.1,2.1,6.4 \\
14[0.1]\end{array}$ \\
\hline 1,2,3,6,7,8-HxCDF & $\begin{array}{c}14.4(2.2) \\
3.5,8.7,49.1 \\
2[0.1] \\
\end{array}$ & $\begin{array}{c}23.3(7.8) \\
12.7,17.8,90.8 \\
0 \text { [NA] }\end{array}$ & $\begin{array}{c}14.5(9.7) \\
1.2,3.2,27.8 \\
4[0.1]\end{array}$ & $\begin{array}{c}3.9(0.5) \\
1.8,4.2,13.4 \\
2[<0.05]\end{array}$ & $\begin{array}{c}2.4(0.3) \\
1.2,2.4,10.2 \\
6(0.2)\end{array}$ \\
\hline 1,2,3,7,8,9-HxCDF & $\begin{array}{c}10.2(1.7) \\
1.3,5.3,37.5 \\
22[0.3]\end{array}$ & $\begin{array}{c}9.5(5.3) \\
3.1,5.8,31.1 \\
0[\mathrm{NA}]\end{array}$ & $\begin{array}{c}11.6(9.0) \\
0.3,1.3,22.6 \\
41[0.2]\end{array}$ & $\begin{array}{c}1.7(0.3) \\
0.4,1.0,9.7 \\
39[0.1]\end{array}$ & $\begin{array}{c}0.4(0.1) \\
0.2,0.5,1.1 \\
94[0.1]\end{array}$ \\
\hline 2,3,4,6,7,8-HxCDF & $\begin{array}{c}8.1(1.3) \\
2.3,5.8,34.7 \\
3[0.1]\end{array}$ & $\begin{array}{c}15.4(5.0) \\
7.2,12.8,44.6 \\
0[\mathrm{NA}]\end{array}$ & $\begin{array}{c}7.0(4.6) \\
0.8,1.8,13.9 \\
2[0.4]\end{array}$ & $\begin{array}{c}2.7(0.3) \\
1.3,3.1,10.7 \\
1[0.3]\end{array}$ & $\begin{array}{c}1.9(0.2) \\
1.0,2.5,6.5 \\
17[0.2]\end{array}$ \\
\hline 1,2,3,4,6,7,8-HpCDF & $\begin{array}{c}88.5(17.5) \\
26.4,70.9,272 \\
0[\mathrm{NA}]\end{array}$ & $\begin{array}{c}221.2(45.8) \\
144,225,766 \\
0 \text { [NA] }\end{array}$ & $\begin{array}{c}43.6(16.1) \\
8.6,23.6,135 \\
0[\mathrm{NA}]\end{array}$ & $\begin{array}{c}31.3(4.8) \\
15.5,32.4,109 \\
0[\mathrm{NA}]\end{array}$ & $\begin{array}{c}30.1(4.6) \\
11.3,31.1,169 \\
0 \text { [NA] }\end{array}$ \\
\hline 1,2,3,4,7,8,9-HpCDF & $5.6(0.8)$ & $13.2(3.7)$ & $4.3(2.5)$ & $1.9(0.3)$ & $1.1(0.1)$ \\
\hline
\end{tabular}




\begin{tabular}{|c|c|c|c|c|c|}
\hline & $\begin{array}{c}1.6,4.1,16.7 \\
9(0.4)\end{array}$ & $\begin{array}{c}7.9,11.8,53.2 \\
0 \text { [NA] }\end{array}$ & $\begin{array}{c}0.5,1.4,10.4 \\
20[0.3]\end{array}$ & $\begin{array}{c}0.8,2.0,5.5 \\
12[0.2]\end{array}$ & $\begin{array}{c}0.6,1.2,2.9 \\
50[0.2]\end{array}$ \\
\hline OCDF & $\begin{array}{c}132.1(20.1) \\
52.2,105,520 \\
0 \text { [NA] }\end{array}$ & $\begin{array}{c}511.5(111.7) \\
300,497,1370 \\
0[\mathrm{NA}] \\
\end{array}$ & $\begin{array}{c}83.2(31.3) \\
14.7,46.0,207 \\
0 \text { [NA] }\end{array}$ & $\begin{array}{c}66.6(10.2) \\
29.2,72.4,217 \\
0 \text { [NA] } \\
\end{array}$ & $\begin{array}{c}57.7(10.7) \\
21.7,60.0,189 \\
0 \text { [NA] }\end{array}$ \\
\hline PCB 77 & $\begin{array}{c}46.9(9.9) \\
18.5,31.1,110 \\
0 \text { [NA] }\end{array}$ & $\begin{array}{c}221.4(120.1) \\
55.4,138,2210 \\
0[\mathrm{NA}]\end{array}$ & $\begin{array}{c}35.7(18.0) \\
7.6,17.9,63.2 \\
0 \text { [NA] }\end{array}$ & $\begin{array}{c}38.0(4.7) \\
13.7,43.9,128 \\
0[\mathrm{NA}]\end{array}$ & $\begin{array}{c}79.0(32.4) \\
13.7,38.1,154.5 \\
0 \text { [NA] }\end{array}$ \\
\hline PCB 81 & $\begin{array}{c}2.8(0.5) \\
1.2,2.5,5.9 \\
15[0.4]\end{array}$ & $\begin{array}{c}8.5(3.0) \\
3.1,8.3,56.7 \\
0[\mathrm{NA}]\end{array}$ & $\begin{array}{c}2.8(1.2) \\
0.6,1.2,13.6 \\
25[0.5]\end{array}$ & $\begin{array}{c}2.3(0.3) \\
1.0,2.3,8.8 \\
23[0.3]\end{array}$ & $\begin{array}{c}3.1(1.1) \\
0.8,2.2,6.4 \\
18[0.2]\end{array}$ \\
\hline PCB 126 & $\begin{array}{c}7.2(1.1) \\
4.2,7.1,28.9 \\
2[1.0]\end{array}$ & $\begin{array}{c}51.5(28.3) \\
9.3,23.9,509 \\
0[\mathrm{NA}]\end{array}$ & $\begin{array}{c}3.2(0.4) \\
1.8,3.6,10.5 \\
4[1.3]\end{array}$ & $\begin{array}{c}6.8(0.5) \\
3.8,8.1,21.8 \\
7[0.8]\end{array}$ & $\begin{array}{c}17.9(5.7) \\
4.4,10.1,39.8 \\
5[0.3]\end{array}$ \\
\hline PCB 169 & $\begin{array}{c}1.0(0.2) \\
0.8,1.0,2.8 \\
26[0.5]\end{array}$ & $\begin{array}{c}3.9(1.6) \\
1.4,2.4,25.5 \\
6[1.3]\end{array}$ & $\begin{array}{c}0.6(0.0) \\
0.5,0.6,1.1 \\
40[0.5]\end{array}$ & $\begin{array}{c}0.9(0.1) \\
0.6,1.0,2.2 \\
28[0.5]\end{array}$ & $\begin{array}{c}1.0(0.1) \\
0.7,1.2,2.7 \\
50[0.4]\end{array}$ \\
\hline PCB 105 & $\begin{array}{c}365.6(80.7) \\
151,232,1100 \\
0[\mathrm{NA}]\end{array}$ & $\begin{array}{c}5508.9(4317.8) \\
374.5,936,79000 \\
0[\mathrm{NA}]\end{array}$ & $\begin{array}{c}189.1(50.2) \\
48.8,123,843 \\
0[\mathrm{NA}]\end{array}$ & $\begin{array}{c}351.1(34.1) \\
131,335,1430 \\
0[\mathrm{NA}]\end{array}$ & $\begin{array}{c}2373.8(1198.4) \\
139,497,3090 \\
0 \text { [NA] }\end{array}$ \\
\hline PCB 114 & $\begin{array}{c}16.0(3.3) \\
6.7,11.7,53.5 \\
17[0.8]\end{array}$ & $\begin{array}{c}269.5(214.0) \\
17.4,72.7,3915 \\
0 \text { [NA] }\end{array}$ & $\begin{array}{c}11.6(3.7) \\
1.9,6.3,38.8 \\
28[0.9]\end{array}$ & $\begin{array}{c}14.4(1.6) \\
4.9,12.4,57.5 \\
31[0.5]\end{array}$ & $\begin{array}{c}106.8(60.0) \\
4.3,15.5,126 \\
40[0.6]\end{array}$ \\
\hline РCB 118 & $\begin{array}{c}655.1(146.5) \\
265,429,2350 \\
0[\mathrm{NA}]\end{array}$ & $\begin{array}{c}11995(9571) \\
678,1860,175000 \\
0[\mathrm{NA}]\end{array}$ & $\begin{array}{c}365.3(98.9) \\
91.9,240,1920 \\
0[\mathrm{NA}]\end{array}$ & $\begin{array}{c}648.8(69.7) \\
248,565,2500 \\
0[\mathrm{NA}]\end{array}$ & $\begin{array}{c}4982.7(2601.1) \\
244,903,7330 \\
0 \text { [NA] }\end{array}$ \\
\hline РCB 123 & $\begin{array}{c}17.3(3.2) \\
7.8,14.1,54.9 \\
6(0.8)\end{array}$ & $\begin{array}{c}183.3(139.8) \\
16.7,41.3,2560 \\
0[\mathrm{NA}]\end{array}$ & $\begin{array}{c}9.2(2.3) \\
3.0,7.0,32.9 \\
12[1.5]\end{array}$ & $\begin{array}{c}15.0(1.1) \\
7.4,15.2,58.3 \\
11[0.6]\end{array}$ & $\begin{array}{c}87.9(45.1) \\
7.8,22.8,132 \\
13[0.5]\end{array}$ \\
\hline РCB 156 & $\begin{array}{c}100.2(18.7) \\
48.6,87.8,307 \\
0[\mathrm{NA}]\end{array}$ & $\begin{array}{c}1615.9(1213.3) \\
103,303,22200 \\
0[\mathrm{NA}]\end{array}$ & $\begin{array}{c}51.6(12.2) \\
17.3,39.9,217 \\
0[\mathrm{NA}]\end{array}$ & $\begin{array}{c}118.6(14.5) \\
48.8,113,426 \\
1[0.2]\end{array}$ & $\begin{array}{c}755.5(371.6) \\
68.9,189,1200 \\
0 \text { [NA] }\end{array}$ \\
\hline PCB 157 & $\begin{array}{c}25.4(4.6) \\
12.8,21.2,77.0 \\
0[\mathrm{NA}]\end{array}$ & $\begin{array}{c}369.9(283.0) \\
24.2,69.4,5180 \\
0[\mathrm{NA}]\end{array}$ & $\begin{array}{c}12.8(2.9) \\
4.8,10.3,50.7 \\
0[\mathrm{NA}]\end{array}$ & $\begin{array}{c}28.0(3.2) \\
12.6,28.4,97.5 \\
0[\mathrm{NA}]\end{array}$ & $\begin{array}{c}179.1(83.6) \\
15.9,48.8,333 \\
1[0.5]\end{array}$ \\
\hline PCB 167 & $\begin{array}{c}40.8(6.7) \\
20.9,36.2,118 \\
1[1.7]\end{array}$ & $\begin{array}{c}563.7(402.2) \\
38.4,125,7345 \\
0 \text { [NA] }\end{array}$ & $\begin{array}{c}20.8(4.3) \\
7.9,16.7,71.7 \\
1[3.0]\end{array}$ & $\begin{array}{c}45.4(5.1) \\
20.5,47.2,153 \\
0[\mathrm{NA}]\end{array}$ & $\begin{array}{c}271.9(126.5) \\
27.9,82.2,477 \\
0 \text { [NA] }\end{array}$ \\
\hline PCB 189 & $\begin{array}{c}9.5(1.2) \\
4.9,9.2,33.2 \\
3[0.9]\end{array}$ & $\begin{array}{c}86.7(47.3) \\
9.3,29.8,706.5 \\
0 \text { [NA] }\end{array}$ & $\begin{array}{c}3.7(0.5) \\
2.1,4.3,10.6 \\
3[0.3]\end{array}$ & $\begin{array}{c}10.3(1.4) \\
4.6,11.3,29.7 \\
1[0.1]\end{array}$ & $\begin{array}{c}37.8(14.3) \\
6.8,18.3,81.3 \\
8[0.3]\end{array}$ \\
\hline
\end{tabular}

HP = house perimeter; LOD = Limit of detection; M/S = Midland/Saginaw; NA = not applicable. 